\title{
The field-dependent rheological properties of magnetorheological fluids featuring plate-like iron particles
}

\author{
Kruti Shah and Seung-Bok Choi * \\ Smart Structures and Systems Laboratory, Department of Mechanical Engineering, Inha University, Incheon, South Korea
}

Edited by:

Weihua Li, University of Wollongong, Australia

\section{Reviewed by:}

Xufeng Dong, Dalian University of

Technology, China

Xian-Xu Bai, Hefei University of

Technology, China

${ }^{*}$ Correspondence:

Seung-Bok Choi, Smart Structures and Systems Laboratory, Department of Mechanical Engineering, Inha University, Incheon 402-751, South Korea

e-mail:seungbok@inha.ac.kr
This study is concerned with an investigation of the plate-like iron particles based MR suspensions under the application of magnetic fields to ascertain the influence of particle size in the rheological performance. A novel synthesis route to prepare magnetorheological fluid (MRF) using two different sizes of plate-like iron particles is described in detail. Two different kinds of MRF are then prepared and their rheological properties are presented and discussed extensively. Steady-shear flow and small-amplitude dynamic oscillatory measurements are carried out in the presence of magnetic field. This experimental study reveals and highlights the importance of exploiting some parameters such as magnetic field strength, effect of particle size, and magnetoviscous and viscoelastic properties of the suspending fluid. The magnetization of the fluids is also performed to explain the effect of particle size in the magnetic field, which is directly correlated with the yield stress. In the absence of magnetic field, the properties of fluid are isotropic and upon the application of magnetic field the magnetized particles form strong-chain-like structures in the field direction, which promotes the appearance of yield stress. This material is known as smart material whose properties amend from liquid to solid immediately after applying the magnetic field. It is found from this work that the large size particle based MRF exhibits high yield stress and strong-chain structuration under the applying magnetic field.

Keywords: magnetorheological fluid, plate-like iron particles, particle size, Bingham model, yield stress

\section{INTRODUCTION}

Magnetorheological fluids (MRFs) are suspensions that reversibly change their rheological behavior under the magnetic field between liquid and solid-like phenomenon. In the absence of magnetic field, the suspension behaves as a typical colloidal system. In the application of magnetic field, particles are aggregate and form a columnar chain-like structure in the field direction. The resulting field induces yield stress and viscoelasticity promote in rheological measurements in MR performance. The ratio between the material function in the presence of magnetic field and in the absence of magnetic field during rheology measurements is defined as MR effect (Boczkowska and Awietjan, 2012). The key property of MR fluid is yield stress and viscoelasticity. Thus, a number of studies have been done to develop MR materials with enhance MR effect (de Vicente et al., 2011). Foister (1997) reported that the MR suspensions composed of mixtures of magnetizable particles of two different diameters $(1.25$ and $7.9 \mu \mathrm{m})$ can provide a substantial increase in the field-induced yield stress without an increase in the viscosity of the mixture. Tang et al. (2000) have observed that by changing the microstructure of the fluid the yield stress of MRF can be enhanced. They compressed the MR fluid in the field direction after applying magnetic field. They have observed in the presence of the magnetic field the static yield stress increases 10 times higher than that of the same MR fluid without compression. They suggested that the enhanced stress due to the increase in packing density can be also achieved with bidisperse suspensions, coating, or normal pressure. The viscoelastic properties of
MRF have been studied by Claracq et al. (2004). They conducted the study on rheological experiments in dynamic mode at very low strain, which facilities the comprehension on the influence of the structure on MRF rheology. Their results have confirmed that the cohesion of the particles in aggregates and the storage modulus is linked between magnetic forces. The system with smallest deformation is not purely elastic, but it is also dissipated some energy, which is denoted by loss modulus. Rankin et al. (1999) studied on MR effect in viscoplastic media. The steady-shear MR response was independent of the continuous phase for the yield stresses in the range $0.9-37 \mathrm{~Pa}$. The field-induced suspension yield stress increased sub-quadratically with the flux density. They have observed that the storage modulus depends not only on the magnitude of the applied magnetic field but also on its history. This history dependence can be explained in terms of the field-dependent evolution of the suspension microstructure. Viscoelasticity of mono- and polydisperse inverse ferrofluids has been studied by Saldivar-Guerrero et al. (2006). Vicente et al. studied rheological properties in rotational (De Vicente et al., 2010) and oscillatory (De Vicente et al., 2009) regime by using MRFs. They studied rheological properties using three different morphologies in particle shape covering one, two, and three dimensional materials with different volume fractions. They observed two rheological parameters of dynamic yield stress and storage modulus. These properties were increased in the beginning stage due to the structuration of the colloid at lower external fields. Once particle magnetic saturation was fully achieved at large external magnetic 
fields, the dynamic yield stress and storage modulus were larger for rod-based than for sphere and plate-based MR fluids for small and intermediate fields, but the difference became smaller. They assumed that stronger fluids are expected to be prepared using anisotropic particles. On the basis of this argument, in this study, plate-like iron particle based MR fluid has been prepared. It is expected that the formation of less compact anisotropy in iron particles creates weak sedimentation and good redispersibility. Even though some research works on the rheological properties and applications (De Vicente et al., 2010; Shah et al., 2013, 2014a,b; Phu et al., 2014; Upadhyay et al., 2014) of the plate-like iron particles based MR fluids have been undertaken, a solid work on the field-dependent rheological properties under various conditions is considerably rare.

Consequently, the main contribution of this work is to investigate the rheological properties of MRF featuring two different sizes of iron plate-like particles. Plate-like shape provides a strong magnetic structure along its long axis aligned with the external magnetic field. In this work, it is firstly discussed about the synthesis procedure to prepare two different kinds of novel MRFs. Next, structural and morphology of magnetic particles and magnetic properties of MRFs are discussed briefly. Subsequently, the fielddependent rheological properties of the proposed MRF in static and dynamic modes are extensively investigated using the magnetorheometer. The comparative results of the proposed MRF are provided with detail discussions on rheological characterizations in terms of magnetoviscous and viscoelastic properties followed by the conclusion.

\section{EXPERIMENTAL DETAILS MATERIALS AND PREPARATION OF MRFs}

In order to check the influence of particle size on the rheological properties of MR fluid, two different MR suspensions are prepared. MR suspension consists of iron plate-like particles suspended in a hydrocarbon base carrier liquid. Iron micro-sized particles (Industrial Metal Powder, Pune, India) having average particle size $2 \mu \mathrm{m}$ (small size) and $19 \mu \mathrm{m}$ (large size) are used to prepare two different MR fluids, which are labeled as S-MRF and L-MRF, respectively. The particles are fully mixed with the paraffin oil (64cSt) using mechanical stirrer. A small amount of stabilizer is added to improve sedimentary stability due to the large density mismatch of the particle and base oil. In this process, the total particle volume fraction of MRF samples is chosen by $16 \%$. The gradual homogenization of the sample is confirmed by the disappearance of the aggregates initially observed at the bottom of container. The samples are vigorously shaken to ensure the required homogeneity before every measurement.

\section{MAGNETORHEOLOGY CHARACTERIZATION}

The structural properties of the micron-size magnetic particles are characterized by X-ray diffractometer, D2-phaser (Bruker XRD, Germany). A detailed analysis of the pattern is undertaken using a (The Rietveld Refinement Program). The morphology of both the small and large size particles is investigated by using the Scanning Electron Microscope (SEM) technique. The room temperature magnetization measurements are carried out using a home-built magnetometer. The room temperature $(300 \mathrm{~K})$ magnetization curves for the MRF samples are carried out and saturation magnetization is measured. The plate-plate magnetorheometer (Physica MCR 301, Anton Paar, GmbH, Austria) is used to test the static and dynamic rheological properties of proposed MRFs. The rheometer is coupled with a commercial magnetorheological device (MRD $180 / 1 \mathrm{~T}$ magnetorheological cell). A diameter of plate is $20 \mathrm{~mm}$ and a gap between parallel disks is kept to $1 \mathrm{~mm}$ during whole experiment. The magnetic circuit is designed so that the magnetic flux lines are normal to the parallel disk. The MR cell is attached to the circulating water bath to maintain temperature throughout the data during all data recording. In the oscillatory tests, a sinusoidal strain loading is applied to the sample by the rotating plate. The response signals are collected by the torque sensor and processed by RHEOPLUS software. The rheological properties are carried out in static and dynamic mode, with the fluid being stirred at a constant shear rate of $100 \mathrm{~s}^{-1}$, unless specified for $30 \mathrm{~s}$ and then left to be stable for another $30 \mathrm{~s}$ before recording the data. The rheology test is performed at $313 \mathrm{~K}$. The specific measurement procedures for each test are given as follows. (1) Shear Rate Dependency - in the absence and/or presence of the magnetic field, the fluid is homogenized at a constant shear rate $(\dot{\gamma}) 100 \mathrm{~s}^{-1}$ for $30 \mathrm{~s}$. The suspension is then left to be stable for $30 \mathrm{~s}$. The shear rate is varied from 1 to $800 \mathrm{~s}^{-1}$ with the fluid response measured every $30 \mathrm{~s}$. Each data is measured using fresh new sample. (2) Amplitude Sweep - the linear viscoelastic range (LVE) is determined, followed by measuring the storage and loss modulus as a function of strain amplitude at angular frequency $\omega=10 \mathrm{rad} \mathrm{s}^{-1}$, in the presence of a magnetic field for MR samples. The experimental procedure can be summarized as follows: (i) initial preshear at a shear rate of $100 \mathrm{~s}^{-1}$ for $30 \mathrm{~s}$; (ii) suspension is left to stable for $30 \mathrm{~s}$ in the absence of a magnetic field; (iii) strain is increased from 0.01 to $100 \%$ at a rate of six points per decade at a given field. (3) Frequency Sweep - (i) initial preshear at a shear rate of $100 \mathrm{~s}^{-1}$ for $30 \mathrm{~s}$; (ii) suspension is left to stabilize for $30 \mathrm{~s}$ in the absence of magnetic field; (iii) strain is fixed at $0.01 \%$, and the angular frequency is increased from 0.1 to $100 \mathrm{rad} \mathrm{s}^{-1}$ at a rate of five points per decade at different fields. (4) Magneto Sweep Test - constant dynamic mechanical conditions are preset like strain $0.01 \%$ and $\omega=10 \mathrm{rad} \mathrm{s}^{-1}$ keep constant. The magnetic field strength is increased from 0.1 to $159.5 \mathrm{kA} \mathrm{m}^{-1}$. Experiments are repeated with fresh new samples in every case.

\section{RESULTS AND DISCUSSIONS MORPHOLOGY OF SYNTHESIZED MAGNETIC PARTICLES}

The size and surface morphology of the iron plate-like small and large size particles are measured by SEM as shown in Figures 1A,B, respectively. The average size of a small particle is $2 \mu \mathrm{m}$ and a large particle is $19 \mu \mathrm{m}$. The particles are of different shapes and sizes, ranging from 2 to $10 \mu \mathrm{m}$ for the small size and 19 to $26 \mu \mathrm{m}$ for the large size. The micro-tech particle size analyzer d50 size is around $6-7 \mu \mathrm{m}$ for a small and $22-24 \mu \mathrm{m}$ for a large. Figure $1 \mathrm{C}$ shows the X-ray diffraction pattern of plate-like iron particles recorded at room temperature. The patterns reveal the formation of body centered cubic structure having a lattice parameter of $0.2864 \pm 0.0001 \mathrm{~nm}$.

The room temperature $(300 \mathrm{~K})$ magnetization curve for SMRF and L-MRF are presented in Figure 2. It is seen that as the 


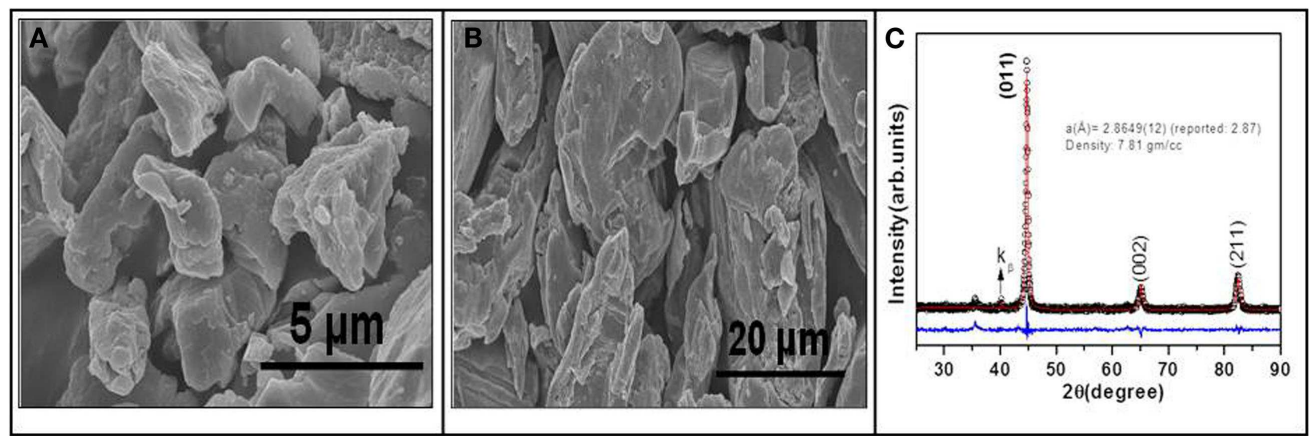

FIGURE 1 | Morphology of (A) the small-sized particle (B) the large sized particle, and (C) structural characterization of plate-like iron particles

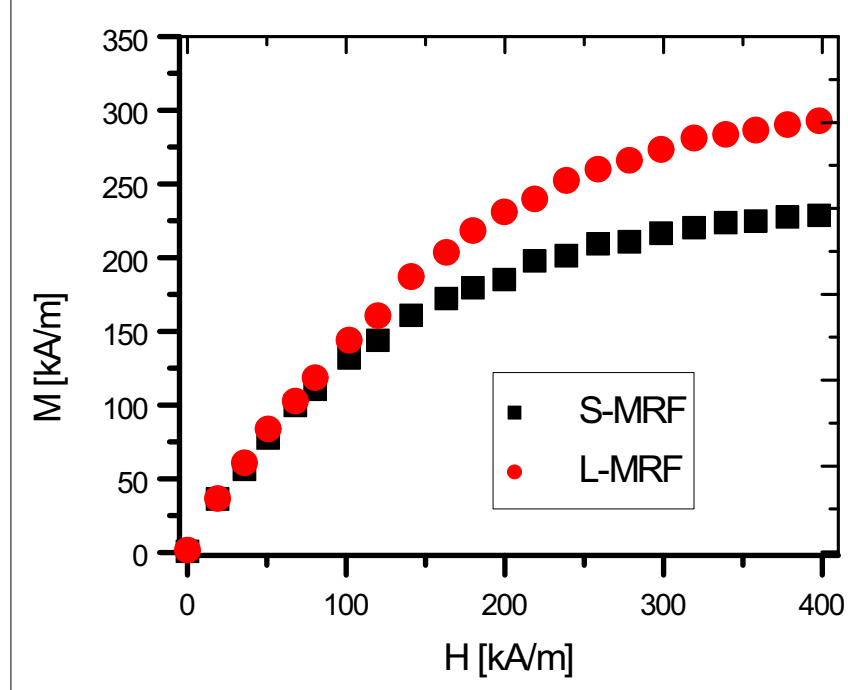

FIGURE 2 | Room temperature (300 K) magnetization measurement of the S-MRF and L-MRF samples is shown.

particle size increases, the magnetization of fluid S-MRF to L-MRF increases from 229 to $293 \mathrm{kA} \mathrm{m}^{-1}$, respectively. In a field gradient, alignment of the magnetic moment of large size particle is faster compared to the small-sized particle. Under this condition, initial susceptibility and saturation magnetization of larger particles is higher compared to the smaller particle.

\section{PROPERTIES UNDER STEADY-SHEAR STATE}

The rheological evolution of the MR suspensions is a useful tool for elucidating the transition from liquid-like to solid-like state under the application of a magnetic field. Figure 3 shows the rheograms corresponding to the S-MRF and L-MRF suspensions for the same volume fraction $16 \%$ and in the absence and maximum applied magnetic field strength $\left(H=228 \mathrm{kA} \mathrm{m}^{-1}\right)$. In the absence and maximum magnetic field strength $\left(H=228 \mathrm{kA} \mathrm{m}^{-1}\right)$ of magnetic field, the viscosity and shear stress as a function of shear rate are shown in Figures 3A,B, respectively.

The measured results show that shear stress and viscosity of S-MRF and L-MRF are same in the absence of magnetic field.
While in the presence of magnetic field we can see that, for a given shear rate the apparent shear stress and viscosity are increased with the diameter of the particles. It is interesting that the rheology of MR fluid is very sensitive to particle size in the presence of magnetic field. The rheological behavior of MRF samples can be better described by the Bingham model than others, since the curve shows a larger linear region. The dynamic yield stress of MR fluids are calculated according to the Bingham plastic model given by Macosko (1994):

$$
\tau=\tau_{B}+\eta_{p} \dot{\gamma}
$$

where, $\tau$ is the shear stress, $\dot{\gamma}$ shear rate, $\tau_{B}$ is the Bingham yield stress, and $\eta_{p}$ is the field-independent plastic viscosity defined as the slope of the shear-stress curve at higher shear rate. It exhibits the yield stress calculated by extrapolation from the Figure 3B considering that the Bingham plastic model is valid. From Eq. 1 , the yield stress of S-MRF and L-MRF is calculated, which increases from 19.30 to $30.98 \mathrm{kPa}$ at maximum applied magnetic field strength $\left(H=228 \mathrm{kA} \mathrm{m}^{-1}\right)$ with augmenting particle size, respectively. Similar results are observed for fluid magnetization, which also increases with augmenting particle size. It seems that there is direct correlation between yield stress, initial susceptibility, and saturation magnetization of fluid.

\section{PROPERTIES UNDER OSCILLATORY SHEAR}

Oscillatory experiments give information on the viscoelastic properties of the proposed MRFs. The rheological properties of MR suspension depend on the deformation history, which created by the microstructure of MRF. Oscillatory tests at sufficiently low deformations do not destroy the structure. This test with small amplitudes can be sensitive to detecting of fine structural changes even on a small scale, which can be evolved during a deformation history (Larson, 1999). As a consequence, the viscoelastic moduli versus deformation is extensively investigated. Furthermore, the magnetic field dependence of the viscoelastic moduli in magneto sweep mode is observed.

In order to study the effect of shear strain on the dynamic viscoelastic moduli of the MRF, an experiment in the strain amplitude sweep mode is carried out. The transition from the solid-like behavior to the liquid-like is similar to gel-sol transition 


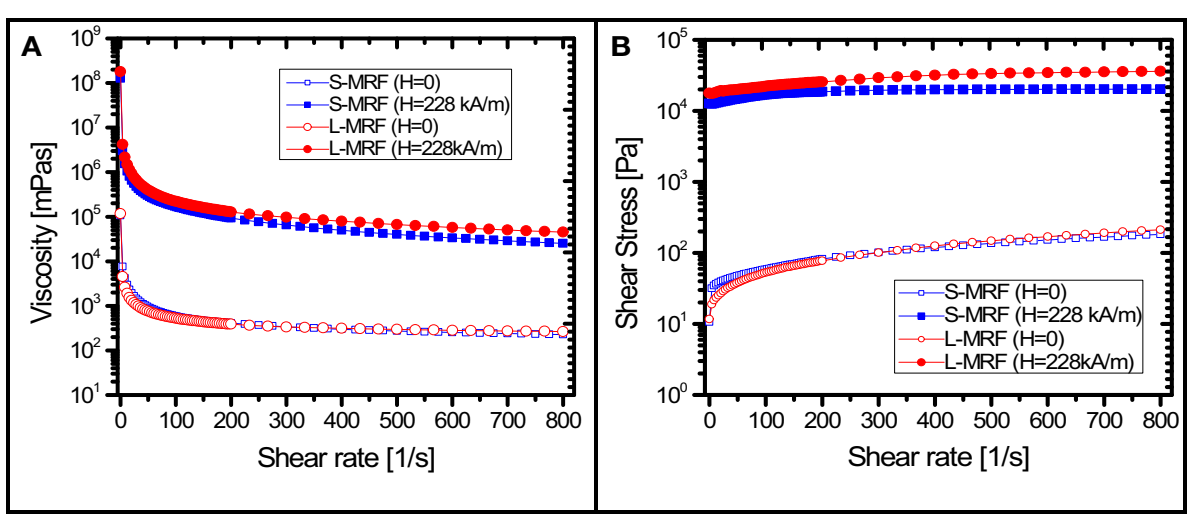

FIGURE 3 | Viscosity (A) and shear stress (B) as a function of shear rate in the absence and presence of maximum magnetic field strength are shown

observed in many gelling materials. Here, strain is the main parameter for the gel-sol transition. The construction and destruction of chain structures are directly related to the strain amplitude sweep. The storage modulus $G^{\prime}$ indicate elastic behavior due to the structuring and recovered the mechanical energy while the loss modulus $G^{\prime \prime}$ indicates viscous behavior due to the dissipation of mechanical energy during the destruction of the elastic structure. Figure 4 shows the plots of storage modulus $G^{\prime}$ and loss modulus $G^{\prime \prime}$ against the strain amplitude $(\gamma)$ at $H=30.97 \mathrm{kA} \mathrm{m}^{-1}$ and frequency $\omega=10 \mathrm{rad} / \mathrm{s}$ for the S-MRF and L-MRF suspensions.

It is seen that at small strain amplitude, storage modulus $G^{\prime}$ and loss modulus $G^{\prime \prime}$ show a strain-independent plateau, which is defined as LVE over in which irreversible destruction of structure begins. After applying magnetic field at small strain amplitude, the randomly dispersed iron particles in fluid are aligned in the field direction and re-assemble to form a strong-chain structuration whose response is linear. However, by increasing the particle size, the height of the $G^{\prime}$ and $G^{\prime \prime}$ plateau decreases and becomes narrow. It indicates that the strain range for the linear viscoelasticity is narrowed by increasing particle size. The strain point where storage modulus $G^{\prime}$ and loss modulus $G^{\prime \prime}$ are equal is known as crossover strain, which signifies the transition between linear viscoelastic solid to the non-linear viscoelastic deformation. Thus, this physical parameter is directly linked to the state of the structure. Interesting results are observed from Figure 4 that with increasing particle size the crossover strain value shifts toward high strain, which indicates L-MRF due to the greater magnetostatic interaction between the large magnetic particles, which have stronger structural strength compared to S-MRF. The longer chains are destroyed greatly and number of shorter chains and free particles are increased, as a result of that, the storage modulus $G^{\prime}$ begins to decrease whereas loss modulus $G^{\prime \prime}$ starts to increase beyond the crossover strain. A complete destruction of chains is occurred at very large strain amplitude.

\section{FREOUENCY SWEEP}

In frequency sweep test, the sample is subjected to a constant strain amplitude define from the LVR by varying the oscillatory frequency. The rheological response of the proposed S-MRF and L-MRF under frequency sweep test is shown in Figure 5. In a

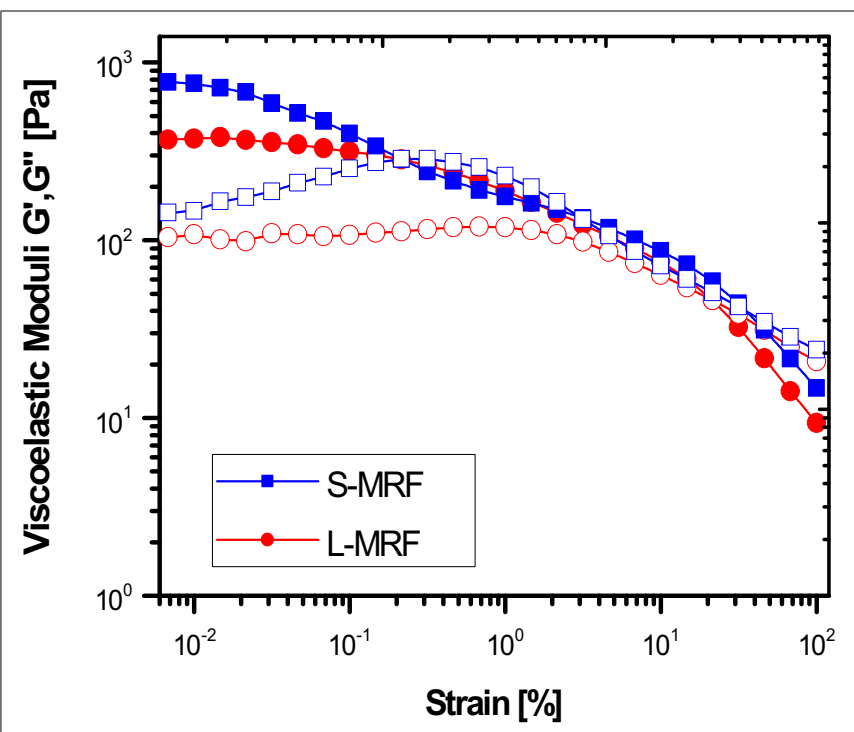

FIGURE 4 | Amplitude sweep test for S-MRF and L-MRF samples at magnetic field strength $H=30.97 \mathrm{kA} \mathrm{m}^{-1}$ (filled symbol $G^{\prime}$, open symbol $G^{\prime \prime}$.

frequency sweep mode at small strain amplitude $\gamma_{0}=0.01 \%$, the effect of particle size on the viscoelastic moduli is examined at different magnetic field strength and presented in Figures 5A,B for S-MRF and L-MRF samples, respectively. It is shown that the storage modulus increases with rising magnetic field strength in MRFs. The storage modulus $G^{\prime}$ characterizing the elastic component of MRFs mainly results from the magnetostatic forces between particles and chains. In this situation, the hydrodynamic forces are unimportant compared to particle-particle or chain-chain interactions, which form into microstructure (Cutillas and Liu, 2002). Therefore, $G^{\prime}$ remains constant at small range of frequency. A slight change in the magnitude of storage modulus with increasing magnetic field and particle size may be attributed to maintenance of magnetized particles chain formation. The loss modulus $G^{\prime \prime}$ is related to the storage modulus, but the interpretation of loss modulus is complicated. At low frequency, loss modulus $G^{\prime \prime}$ is slightly 


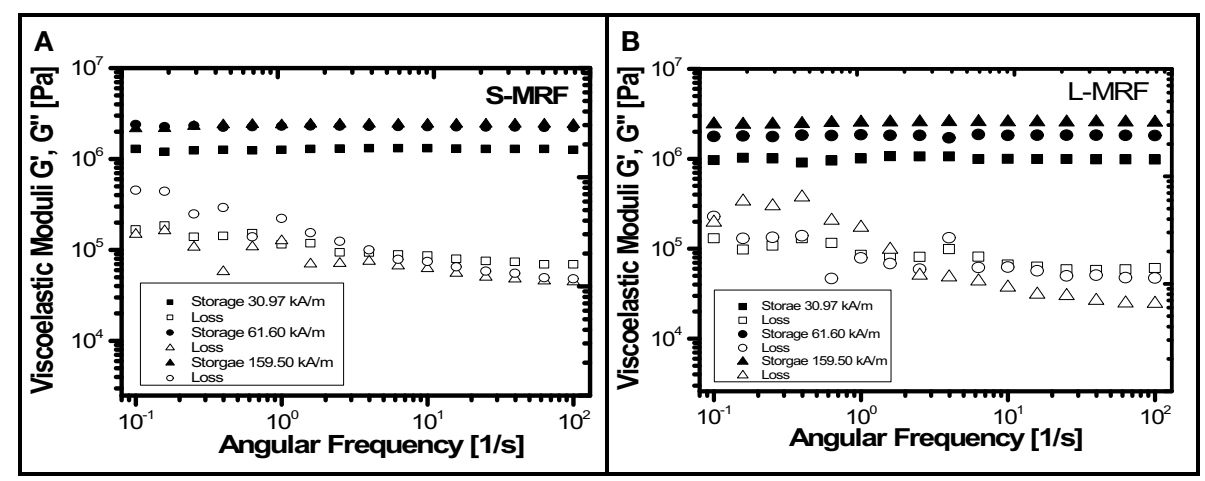

FIGURE 5 | Frequency sweep test for (A) S-MRF and (B) L-MRF samples under the application of magnetic field (filled symbol $G^{\prime}$, open symbol $G^{\prime \prime}$ ) is shown.



FIGURE 6 | Magnetic field dependence of (A) storage modulus $G^{\prime}$, (B) loss modulus $G^{\prime \prime}$, and (C) complex viscosity for S-MRF and L-MRF samples is shown.

different while at high frequency, the superposition overlap takes place. Form Figure 5, it is clear that the loss modulus is dependent on the parameters such as particle size, magnetic field strength, and the range of frequency. At a given magnetic field strength, with increasing frequency, the internal movement of aggregates and relative movement of particles can create some friction between two particles surfaces, which reflects on the loss modulus.

The magneto sweep test can be performed under the application of magnetic field for price measurement of flow ability and structural strength of MRF. Small-amplitude oscillatory shear magneto sweep test is performed at a constant strain amplitude $\gamma_{0}=0.01 \%$ and angular frequency at $\omega=10 \mathrm{rad} / \mathrm{s}$ for S-MRF and L-MRF samples. Figures 6A-C show the storage modulus, loss modulus, and complex viscosity as a function of magnetic field strength, respectively.

At low magnetic field, the difference in flow curve is observed in both samples. At low magnetic field strength, the storage modulus, loss modulus, and complex viscosity decrease with increasing particle size and it behaves as Newtonian fluid. It shows $H^{2}$ dependence behavior in oscillatory test that the structures of magnetic particles become dissolved and more distributed at low magnetic field strength. As a consequence, the influence of small size particle in rheological properties is higher compared to large size, which observed from the height of modulus of the S-MRF, which compared to L-MRF at low magnetic field. Intermediate magnetic field strength, maximum change in both the modulus, and complex viscosity are marked as the transition from viscoelastic liquid to viscoelastic solid behavior. It is known as linear range of chain formation, which becomes linear in the log/log scaled diagram. It shows the $H$-dependency, and the chain-like structure predominates over the base liquid. It shows the storage modulus $G^{\prime}$ increases more than the loss modulus $G^{\prime \prime}$ when the magnetic field strength is increased. This occurs due to the orientation of iron plate-like particles in the field direction. A further increase of the magnetic field strength results in a rigid chain formation in the field direction. This rigid chain formation can prevent the flow and it approaches to saturation in S-MRF and L-MRF samples.

\section{CONCLUSION}

This work presented a detailed experimental study on the magnetoviscous and viscoelastic properties of two different kinds of plate-like iron particles based MRF. MR suspensions have been prepared by using two different sizes of particles disperse in base liquid. The volume fraction of MRF samples has been fixed for both suspensions, which can help to study the influence of particle 
size on rheological properties of MRF in static and dynamic mode. From the magnetization measurements, it has been clearly observed that initial susceptibility and saturation magnetization of large size particle based MRF (L-MRF) are higher compared to small size particle based MRF (S-MRF), which is important to analyze rheology data in static mode. In the presence of magnetic field, the yield stress and viscosity augmenting with increasing particle size directly indicates correlation with the magnetization of fluid. Several properties of the proposed MRF samples have been investigated from dynamic measurements. In strain amplitude sweep test at a given magnetic field, with increasing particle size, the height of the storage modulus $G^{\prime}$ and loss modulus $G^{\prime \prime}$ plateau is decreased and become narrow, but the crossover strain value shifts toward high strain, which indicates stronger structural strength of the L-MRF compared to S-MRF. In frequency sweep test, the storage modulus $G^{\prime}$ increases with rising magnetic field in both MRF samples while due to the fluctuation, it is difficult to interpret the loss modulus. The fluctuations in the loss modulus are related to the storage modulus. The magneto sweep measurement exhibits a strong structuration in their modulus and complex viscosity at high magnetic field strength where the rigid chain formation takes place. The magnetoviscous and viscoelastic study of these fluids shows that the large size particle based fluid (L-MRF) exhibits high yield stress and strong-chain structuration under the applying magnetic field. The strong-chain formation due to the inter particle interaction under the application of magnetic field shows the practical feasibility of the proposed MRF.

\section{ACKNOWLEDGMENTS}

This work was supported by Inha University research grant associated with BK21 Plus Project directed by the National Research Foundation of Korea (NRF) and partially supported by NRF grant funded by the Korea government (MEST) (No. 2010-0015090).

\section{REFERENCES}

Boczkowska, A., and Awietjan, S. (2012). "Microstructure and properties of magnetorheological elastomers," in Advanced Elastomers-Technology, Properties and Applications, ed. A. Boczkowska (Croatia: InTech), 147-180.

Claracq, J., Sarrazin, J., and Montfort, J.-P. (2004). Viscoelastic properties of magnetorheological fluids. Rheol. Acta 43, 38-49. doi:10.1007/s00397-003-0318-7

Cutillas, S., and Liu, J. (2002). Particle dynamics of structure formation and disintegration in a model magnetorheological fluid. Int. J. Mod. Phys. B 16, 2314-2320. doi:10.1142/S0217979202012293

De Vicente, J., Fernando, V., Segovia-Gutierrez, J. P., Morales, M., del, P., and HidalgoAlvarez, R. (2010). Effect of particle shape in magnetorheology. J. Rheol. 54, 1337-1362. doi:10.1122/1.3479045 de Vicente, J., Klingenberg, J. D., and Hidalgo-Alvarez, R. (2011). Magnetorheological fluids: a review. Soft Matter 7, 3701-3710. doi:10.1002/cphc.200900091

De Vicente, J., Segovia-Gutierrez, J. P., Andablo-Reyes, E., Vereda, F., and HidalgoAlvarez, R. (2009). Dynamic rheology of sphere- and rod-based magnetorheological fluids. J. Chem. Phys. 131, 194902. doi:10.1063/1.3259358

Foister, R. T. (1997). Magnetorheological Fluids. US Patent Specification 5667715.

Larson, R. G. (1999). The Structure and Rheology of Complex Fluids. New York: Oxford University Press.

Macosko, C. W. (1994). Rheology: Principles, Measurements, and Applications. New York, NY: VCH.

Phu, D. X., Shah, K., and Choi, S. B. (2014). Design of a new adaptive fuzzy controller and its implementation for the damping force control of a magnetorheological damper. Smart Mater. Struct. 23, 065012. doi:10.1088/0964-1726/23/6/065012

Rankin, P. J., Horvath, A. T., and Klingenberg, D. J. (1999). Magnetorheology in viscoplastic media. Rheol. Acta 38, 471-477. doi:10.1007/s003970050198

Saldivar-Guerrero, R., Richter, R., Rehberg, I., Aksel, N., Heymann, L., and Rodriguez-Fernández, O. S. (2006). Viscoelasticity of mono- and polydisperse inverse ferrofluids. J. Chem. Phys. 125, 084907. doi:10.1063/1.2337576

Shah, K., Oh, J. S., Choi, S. B., and Upadhyay, R. V. (2013). Plate-like iron particles based bidisperse magnetorheological fluid. J. Appl. Phys. 114, 213904. doi:10.1063/1.4837660

Shah, K., Phu, D. X., Seong, M. S., Upadhyay, R. V., and Choi, S. B. (2014a) A low sedimentation magnetorheological fluid based on plate-like iron particles and verification using a damper test. Smart Mater. Struct. 23, 027001. doi:10.1088/0964-1726/23/2/027001

Shah, K., Phu, D. X., and Choi, S. B. (2014b). Rheological properties of bi-dispersed magnetorheological fluids based on plate-like iron particles with application to a small-sized damper. J. Appl. Phys. 115, 203907. doi:10.1063/1.4879681

Tang, X., Zhang, X., Tao, R., and Rong, Y. M. (2000). Structure-enhanced yield stress of magnetorheological fluids. J. Appl. Phys. 87, 2634-2638. doi:10.1063/1.372229

The Rietveld Refinement Program-DBWS-9006PC, developed by Dr H Marciniat, Poland, for X-ray powder and neutron powder diffraction data.

Upadhyay, R. V., Laherisheth, Z., and Shah, K. (2014). Rheological properties of soft magnetic flake shaped iron particle based magnetorheological fluid in dynamic mode. Smart Mater. Struct. 23, 015002. doi:10.1088/0964-1726/23/1/015002

Conflict of Interest Statement: The authors declare that the research was conducted in the absence of any commercial or financial relationships that could be construed as a potential conflict of interest.

Received: 01 September 2014; accepted: 29 September 2014; published online: 15 October 2014.

Citation: Shah K and Choi S-B (2014) The field-dependent rheological properties of magnetorheological fluids featuring plate-like iron particles. Front. Mater. 1:21. doi: 10.3389/fmats.2014.00021

This article was submitted to Smart Materials, a section of the journal Frontiers in Materials.

Copyright (c) 2014 Shah and Choi. This is an open-access article distributed under the terms of the Creative Commons Attribution License (CC BY). The use, distribution or reproduction in other forums is permitted, provided the original author(s) or licensor are credited and that the original publication in this journal is cited, in accordance with accepted academic practice. No use, distribution or reproduction is permitted which does not comply with these terms. 